\title{
Was können, dürfen und wollen wir wissen?
}

\author{
Erhard Taverna \\ Dr. med., Mitglied der Redaktion
}

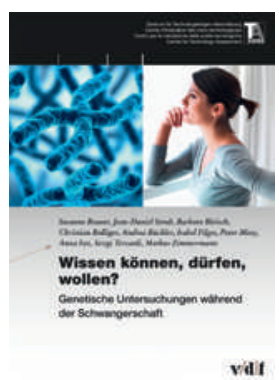

Susanne Brauer, Jean-Daniel Strub, Barbara Bleisch et al. Wissen können, dürfen, wollen?

Genetische Untersuchungen während der Schwangerschaft. Zürich: vdf Hochschulverlag AG an der ETH Zürich; 2016. 452 Seiten. 48 CHF. ISBN 978-3-7281-3748-7
Genetische Untersuchungen während der Schwangerschaft. Eine Studie zur medizinischen Praxis, zu sozialwissenschaftlichen Befunden und ökonomischen Überlegungen im ersten Teil, normativ rechtlich und ethisch beleuchtet in einem zweiten Teil. Zehn Autoren und Autorinnen setzen sich im Auftrag des Zentrums für Technologiefolgen-Abschätzung (TA-SWISS) mit einem brandaktuellen Thema auseinander. Die umfangreiche Auslegeordnung bietet eine exzellente Einführung in die erwähnten Sachgebiete und schliesst mit Empfehlungen, die eine gesellschaftliche Auseinandersetzung anregen sollen. Inhalt sind neue genomische Verfahren, wie der seit 2012 in der Schweiz zugelassene nichtinvasive pränatale Test (NIPT), unmittelbar betroffen sind rund 94000 Frauen mit einer Geburtenzahl von 85000 Kindern (2014). Darüber hinaus ist das Thema ein Paradebeispiel für die zunehmende Komplexität technischer Entwicklungen, die alle Lebensbereiche gewollt oder ungewollt verändern. Mutationen in rund 4000 der etwa 20000 Gene sind heute als krankheitsverursachend identifiziert. Beispiele für monogene Erkrankungen sind die Zystische Fibrose und Hämoglobinopathien. Gemäss den Registern des EUROCAT-Netzwerks (European Surveillance of Congenital Anomalies) hat von 2000 bis 2012 die Zahl der Schwangerschaften mit Trisomie 21, aufgrund älterer Mütter, zugenommen. Die konzentrierte $\mathrm{Zu}$ sammenfassung der medizinischen Diagnostik wagt eine Prognose für die nächsten fünf und zehn Jahre. Der NIPT wird zuverlässiger und billiger. Neue Quellen fetaler DNS, Verfahren wie die Hochfrequenzsequenzierung und der Aufbau bioinformatischer Strukturen werden die Problematik der Zufallsbefunde, zum Beispiel von Tumorprädispositionen, verstärken. Sozialwissenschaftlich steht das Handeln schwangerer Frauen im Mittelpunkt. Einerseits wird die Selbstbestimmung der Frau betont, andererseits ein enormer Informationsbedarf bei gleichzeitig grossen soziokulturellen Unterschieden festgestellt. Das Risiko einer zunehmenden Routinisierung erhöht den Rechtfertigungsdruck für die Schwangeren. Aus ökonomischer Sicht ist der individuelle Nutzengewinn für schwan- gere Frauen schwierig zu beziffern. Die meisten Eltern wünschen Sicherheit und möchten ein Kind ohne Trisomie 21 zur Welt bringen. Verschiedene ScreeningSzenarien zeigen, dass der Einsatz von NIPT als Zweitscreening aus dieser Sicht am wirtschaftlichsten ist. Die Biomedizin-Konvention erlaubt Interventionen im Gesundheitsbereich nur unter der Voraussetzung, dass die betroffene Person aufgeklärt wurde und eine informierte Entscheidung getroffen hat. Die reproduktive Selbstbestimmung stellt hohe Ansprüche an die Beratungspraxis. Im englischen Sprachraum ist der Masterstudiengang des Genetic Counselor weit verbreitet, in Frankreich ist der Studiengang des Conseiller en Génétique seit 2004 anerkannt. Im Zentrum der ethischen Betrachtungen steht die personalisierte Medizin mit Förderung der sogenannten "proaktiven P4-Medizin» (prädiktiv, präventiv, personalisiert, partizipatorisch), die Diskussion über "unwertes Leben» und die damit verbundene Diskriminierung Behinderter sowie eine falsch verstandene Gesundheitsfixierung mit ihrer Orientierung an Norm-Menschen. Ein weiterer Punkt sind mögliche Konflikte zwischen dem elterlichen Recht auf Selbstbestimmung und der elterlichen Pflicht gegenüber dem werdenden Kind sowie dessen moralische Rechte.

Die Projektgruppe vertritt bewusst ein breites Meinungsspektrum. Eine zentrale Empfehlung gilt personell, finanziell und fachlich der umfassenden und qualitativ hochstehenden Aufklärung und Beratung. Das Recht auf Nichtwissen der Schwangeren und die Vorbeugung selektiver Schwangerschaftsabbrüche ohne Notlage (z.B. bei unerwünschtem Geschlecht) sowie Begleitforschung sind weitere wichtige Anliegen von rund vierzehn abschliessenden Vorschlägen an die Ärzteschaft, medizinische Fachgesellschaften, Gesetzgeber, Gesundheitspolitiker und die Expertenkommission für genetische Untersuchungen beim Menschen (GUMEK). Ein ausführliches Glossar erleichtert dem Nichtspezialisten das Verständnis.

Das Kind als Projekt ist eine Überforderung. Auch das wäre eine Schlussfolgerung. 